Korean J. Math. 19 (2011), No. 3, pp. 255-261

\title{
SEMIGROUP RINGS AS H-DOMAINS
}

\author{
Gyu Whan Chang
}

\begin{abstract}
Let $D$ be an integral domain, $S$ be a torsion-free grading monoid such that the quotient group of $S$ is of type $(0,0,0, \ldots)$, and $D[S]$ be the semigroup ring of $S$ over $D$. We show that $D[S]$ is an $\mathrm{H}$-domain if and only if $D$ is an H-domain and each maximal $t$-ideal of $S$ is a $v$-ideal. We also show that if $\mathbb{R}$ is the field of real numbers and if $\Gamma$ is the additive group of rational numbers, then $\mathbb{R}[\Gamma]$ is not an H-domain.
\end{abstract}

\section{Introduction}

Let $D$ be an integral domain with quotient field $K, S$ be a torsionfree grading monoid, and $D[S]$ be the semigroup ring of $S$ over $D$. A $D$-submodule $F$ of $K$ is called a fractional ideal of $D$ if $d F \subseteq D$ for some nonzero $d \in D$. For a nonzero fractional ideal $I$ of $D$, let $I^{-1}=\{x \in$ $K \mid x I \subseteq D\}$; so $I^{-1}$ is also a nonzero fractional ideal of $D$.

1.1. Motivation and Result. An integral domain $D$ is called an $H$ domain if $I$ is an ideal of $D$ such that $I^{-1}=D$, then there is a finitely generated subideal $J$ of $I$ such that $J^{-1}=D$. In [6], Glaz and Vasconcelos introduced the notion of an H-domain, and they then proved that a completely integrally closed H-domain is a Krull domain. They also proved that if $D$ is an H-domain, then $D[X]$, the polynomial ring over $D$, is also an H-domain. Let $\left\{X_{\alpha}\right\}$ be a nonempty set of indeterminates over $D$. Park showed that if $D$ is an H-domain, then so is $D\left[\left\{X_{\alpha}\right\}\right][10$, Proposition 4.2]. Park also showed that if $D$ is a strong Mori domain and if $G$ is a torsion-free abelian group of type $(0,0,0, \cdots)$, then $D[G]$

Received May 12, 2011. Revised August 2, 2011. Accepted August 5, 2011.

2000 Mathematics Subject Classification: 13A15, 20M14.

Key words and phrases: semigroup ring, torsion-free grading monoid, H-domain.

This research was supported by Basic Science Research Program through the National Research Foundation of Korea(NRF) funded by the Ministry of Education, Science and Technology(2010-0007069). 
is an H-domain [10, Proposition 5.5]. Clearly, a strong Mori domain is an H-domain. So it is natural to ask if $D[G]$ is an H-domain when $D$ is an H-domain.

In this paper, we study when $D[S]$ is an H-domain. Precisely, we show that if the quotient group of $S$ is of type $(0,0,0, \ldots)$, then $D[S]$ is an H-domain if and only if $D$ is an H-domain and each maximal $t$ ideal of $S$ is a $v$-ideal. Let $\mathbb{R}$ be the field of real numbers and let $\Gamma$ be the additive group of rational numbers. We prove that $\mathbb{R}[\Gamma]$ is not an H-domain, which shows that the assumption that the quotient group of $S$ is of type $(0,0,0, \ldots)$ is necessary for $D[S]$ to be an H-domain.

1.2. Definition and Notation. Let $\mathbf{F}(D)$ be the set of nonzero fractional ideals of $D$. For each $I \in \mathbf{F}(D)$, let $I_{v}=\left(I^{-1}\right)^{-1}, I_{t}=\cup\left\{J_{v} \mid J \subseteq I\right.$ and $J$ is a nonzero finitely generated ideal $\}$, and $I_{w}=\{x \in K \mid x J \subseteq I$ for some nonzero finitely generated ideal $J$ with $\left.J^{-1}=D\right\}$. An $I \in \mathbf{F}(D)$ is called a $*$-ideal if $I_{*}=I$, where $*=v$, $t$, or $w$, while a $*$-ideal of $D$ is a maximal $*$-ideal if it is maximal among proper integral $*$-ideals of $D$. It is well known that a prime ideal minimal over a $t$-ideal is a $t$-ideal; a maximal $t$-ideal is a prime ideal; and each proper integral $t$-ideal is contained in a maximal $t$-ideal. An integral domain $D$ is a strong Mori domain if $D$ satisfies the ascending chain condition on integral $w$-ideals. In particular, Noetherian domains are strong Mori domains.

Let $S$ be a torsion-free grading monoid with quotient group $G$. It is well known that $D[S]$ is an integral domain [4, Theorem 8.1] and $S$ admits a total order $<$ compatible with its monoid operation [4, Corollary 3.4]. Hence each $f \in D[S]$ is uniquely written in the form

$$
f=a_{0} X^{\alpha_{0}}+a_{1} X^{\alpha_{1}}+\cdots+a_{n} X^{\alpha_{n}},
$$

where $a_{i} \in D$ and $\alpha_{j} \in S$ with $\alpha_{0}<\alpha_{1}<\cdots<\alpha_{n}$. For any $f \in K[G]$, we denote by $A_{f}$ (resp., $E_{f}$ ) the fractional ideal of $D$ (resp., $S$ ) generated by the coefficients (resp., exponents) of $f$; hence $A_{f}=\left(a_{0}, a_{1}, \ldots, a_{n}\right)$ and $E_{f}=\left(\alpha_{0}+S\right) \cup\left(\alpha_{1}+S\right) \cup \cdots \cup\left(\alpha_{n}+S\right)$. The torsion-free abelian group $G$ is said to be of type $(0,0,0, \ldots)$ if $G$ satisfies the ascending chain condition on cyclic subgroups. As in the domain case, one can define the $v$ - and $t$-operation on $S$; and maximal $t$-ideals of $S$.

The reader can refer to $[3, \S 32$ and $\S 34]$ for the $v$-and $t$-operation on integral domains; to $[4, \S 16]$ or [7] for the $v$-and $t$-operation on monoids; and to $[4,7]$ for monoids and monoid domains. 


\section{H-domains}

Let $D$ be an integral domain with quotient field $K, S$ be a torsion-free grading monoid with quotient group $G$ and $D[S]$ be the semigroup ring of $S$ over $D$.

We begin this section with some equivalence conditions of an $\mathrm{H}$ domain. These are already known [8, Proposition 2.4], but we give the proof for the reader's convenience.

LEMMA 1. The following statements are equivalent.

(1) $D$ is an H-domain.

(2) Every maximal $t$-ideal of $D$ is a $v$-ideal.

(3) $I_{v} \subsetneq D$ for each proper integral t-ideal $I$ of $D$.

Proof. (1) $\Rightarrow(2)$ Let $Q$ be a maximal $t$-ideal of $D$. If $Q^{-1}=D$, then there is a nonzero finitely generated ideal $J \subseteq Q$ such that $J^{-1}=D$. So $D=J_{v} \subseteq Q_{t}=Q \subsetneq D$, a contradiction. Hence $Q^{-1} \supsetneq D$, and since $Q$ is a maximal $t$-ideal, $Q=Q_{v}$. (2) $\Rightarrow$ (3) If $I=I_{t} \subsetneq D$, then there is a maximal $t$-ideal $Q$ of $D$ such that $I \subseteq Q$. Hence $I_{v} \subseteq Q_{v}=Q \subsetneq D$. (3) $\Rightarrow(1)$ Let $I$ be a nozero ideal of $D$ with $I^{-1}=D$. Then $I_{v}=D$, and hence $I_{t}=D$ by (3). So there is a finitely generated ideal $J \subseteq I$ such that $J_{v}=D$ or $J^{-1}=D$.

LEMMA 2. Let $S$ be a torsion-free grading monoid with quotient group $G, K$ be a field, $Q$ be a maximal $t$-ideal of $K[S]$, and $N=\left\{X^{\alpha} \mid \alpha \in S\right\}$.

(1) If $Q \cap N \neq \emptyset$, then $J=\left\{\alpha \in S \mid X^{\alpha} \in Q\right\}$ is a maximal $t$-ideal of $S$ and $Q=K[J]$.

(2) If $Q \cap N=\emptyset$, then $Q K[G]$ is a maximal $t$-ideal of $K[G]$.

(3) If $G$ is of type $(0,0,0, \ldots)$ and if $Q \cap N=\emptyset$, then $Q$ is a height-one $t$-invertible prime $t$-ideal.

Proof. (1) [1, Corollary 1.3].

(2) Suppose that $(Q K[G])_{t}=K[G]$. Note that $K[S]_{N}=K[G]$. Then there is a finitely generated subideal $A$ of $Q$ such that $A^{-1} \subseteq A^{-1} K[G]=$ $(A K[G])^{-1}=K[G]\left[11\right.$, Lemma 1.4]. Note that $Q \subsetneq K\left[\cup_{f \in Q} E_{f}\right]$ and $Q$ is a maximal $t$-ideal of $K[S]$. So $\left(\cup_{f \in Q} E_{f}\right)_{t}=S$ (cf. [2, Lemma $2.3]$ ), and hence $\left(E_{f_{1}} \cup \cdots \cup E_{f_{k}}\right)_{v}=S$ for some $f_{1}, \ldots, f_{k} \in Q$. Let $I=\left(A, f_{1}, \ldots, f_{k}\right)$. Then $I$ is a finitely generated subideal of $Q$ such that $I^{-1} \subseteq I^{-1} K[G]=(I K[G])^{-1} \subseteq(A K[G])^{-1}=K[G]$. Let $0 \neq g \in I^{-1}$. Then $g f_{i} \in K[S]$ for $i=1, \ldots, k$ and $K[S]=K\left[\left(\left(m_{1}+1\right) E_{f_{1}} \cup \cdots \cup\right.\right.$ 
$\left.\left.\left(m_{k}+1\right) E_{f_{k}}\right)_{t}\right]=\left(K\left[\left(m_{1}+1\right) E_{f_{1}} \cup \cdots \cup\left(m_{k}+1\right) E_{f_{k}}\right]\right)_{t}[2$, Lemma 2.3], and hence $K\left[E_{g}\right]=\left(K\left[\left(m_{1}+1\right) E_{f_{1}} \cup \cdots \cup\left(m_{k}+1\right) E_{f_{k}}\right] K\left[E_{g}\right]\right)_{t}=$ $\left(K\left[\left(m_{1} E_{f_{1}}+E_{f_{1} g}\right) \cup \cdots \cup\left(m_{k} E_{f_{k}}+E_{f_{k} g}\right)\right]\right)_{t} \subseteq K[S]$ for some positive integers $m_{i}$ [5, Proposition 6.2]; so $g \in K[S]$. Thus $I^{-1}=K[S]$ and $I_{v} \subseteq Q \subsetneq K[S]$, a contradiction. Thus $(Q K[G])_{t} \subsetneq K[G]$. If $Q_{0}$ is a prime ideal of $K[S]$ such that $Q K[G] \subseteq Q_{0} K[G]$ and $Q_{0} K[G]$ is a maximal $t$-ideal of $K[G]$. Then $Q \subseteq Q_{0} K[G] \cap K[S]=Q_{0}$ and $Q_{0}$ is also a prime $t$-ideal $\left[9\right.$, Lemma 3.17]. Thus, $Q=Q_{0}$ and $Q K[G]=Q_{0} K[G]$.

(3) Note that $K[G]$ is a UFD [4, Theorem 14.15], because $G$ is of type $(0,0,0, \ldots)$. Since $Q K[G]$ is a $t$-ideal of $K[G]$ by $(2)$, we have $Q K[G]=h K[G]$ for some $h \in Q$ and $\operatorname{ht} Q=\operatorname{ht}(Q K[G])=1$. Let $f_{1}, \ldots, f_{k} \in Q$ such that $\left(E_{f_{1}} \cup \cdots \cup E_{f_{k}}\right)_{v}=S$ (see the proof of (2)). Then $\left(f_{1}, \ldots, f_{k}, h\right)_{v}=Q[9$, Proposition 2.8]. Also, since $Q$ is a maximal $t$-ideal, $Q$ is $t$-locally principal. Thus, $Q$ is $t$-invertible [9, Corollary $2.7]$.

Lemma 3. Let $S$ be a torsion-free grading monoid, and let $Q$ be a maximal $t$-ideal of $D[S]$.

(1) If $Q \cap D \neq 0$, then $Q=(Q \cap D) D[S]$ and $Q \cap D$ is a maximal $t$-ideal of $D$.

(2) If $Q \cap D=0$, then $Q K[S]$ is a maximal t-ideal of $K[S]$.

Proof. (1) [1, Corollary 1.3].

(2) Note that $D[S]_{D \backslash\{0\}}=K[S]$. If $(Q K[S])_{t}=K[S]$, then there exists a finitely generated ideal $B \subseteq Q$ such that $B^{-1} \subseteq B^{-1} K[S]=$ $(B K[S])^{-1}=K[S][11$, Lemma 1.4]. Since $Q$ is a maximal $t$-ideal of $D[S]$ with $Q \cap D=0,\left(\sum_{g \in Q} A_{g}\right)_{t}=D$, and hence $\left(A_{g_{1}}+\cdots+A_{g_{m}}\right)_{v}=D$ for some $g_{1}, \ldots, g_{m} \in Q$. Let $J=\left(B, g_{1}, \ldots, g_{m}\right)$. Then $J$ is a finitely generated subideal of $Q$ such that $J^{-1} \subseteq J^{-1} K[S]=(J K[S])^{-1} \subseteq$ $(B K[S])^{-1}=K[S]$. Let $0 \neq h \in J^{-1}$. Then $h g_{i} \in D[S]$ for $i=1, \ldots, m$, and hence $\left.A_{h}[S]=\left(\left(A_{g_{1}}^{k_{1}+1}+\cdots+A_{g_{m}}^{k_{m}+1}\right)[S]\right)\left(A_{h}[S]\right)\right)_{t}=\left(\left(A_{g_{1}}^{k_{1}} A_{g_{1} h}\right)+\right.$ $\left.\cdots+\left(A_{g_{m}}^{k_{m}} A_{g_{m} h}\right)[S]\right)_{t} \subseteq D[S]$ for some positive integers $k_{i}$ ([5, Theorem 4.3] and [2, Lemma 2.3]); so $h \in K[S]$. Thus $J^{-1}=D[S]$ and $J_{v} \subseteq Q \subsetneq$ $D[S]$, a contradiction. Thus $(Q K[S])_{t} \subsetneq K[S]$. If $Q^{\prime}$ is a prime ideal of $K[S]$ such that $Q K[S] \subseteq Q^{\prime} K[S]$ and $Q^{\prime} K[S]$ is a maximal $t$-ideal of $K[S]$. Then $Q \subseteq Q^{\prime} K[S] \cap D[S]=Q^{\prime}$ and $Q^{\prime}$ is also a prime $t$-ideal [9, Lemma 3.17]. Thus, $Q=Q^{\prime}$ and $Q K[S]$ is a maximal $t$-ideal. 
LEMma 4. Let $S$ be a torsion-free grading monoid. If $D[S]$ is an $H$-domain, then $D$ is an $H$-domain and every maximal $t$-ideal of $S$ is a $v$-ideal.

Proof. Let $P$ be a maximal $t$-ideal of $D$. Then $P D[S]$ is a prime $t$ ideal of $D[S]$, and hence $P_{v} \subseteq\left(P_{v} D[S]\right)_{v}=(P D[S])_{v} \subsetneq D[S][2$, Lemma 2.3]; so $P_{v} \subsetneq D$. Thus $D$ is an H-domain by Lemma 1 . Let $J$ be a maximal $t$-ideal of $S$. Then $D[J]$ is a $t$-ideal of $D[S]$ [2, Corollary 2.4], and hence $D\left[J_{v}\right]=(D[J])_{v} \subsetneq D[S]\left[2\right.$, Lemma 2.3]. Hence $J_{v} \subsetneq S$, and thus $J=J_{v}$.

THEOREM 5. Let $S$ be a torsion-free grading monoid with quotient group $G$ such that $G$ is of type $(0,0,0, \ldots)$. Then $D[S]$ is an H-domain if and only if $D$ is an H-domain and every maximal t-ideal of $S$ is a $v$-ideal.

Proof. $(\Rightarrow)$ Lemma 4 .

$(\Leftarrow)$ Let $N=\left\{X^{\alpha} \mid \alpha \in S\right\}$ and let $Q$ be a maximal $t$-ideal of $D[S]$. By Lemma 1, it suffices to show that $Q_{v}=Q$.

Case 1. $Q \cap D \neq 0$. Then $Q \cap D$ is a maximal $t$-ideal of $D$ and $Q=(Q \cap D) D[S]$ by Lemma 3(1). Thus $Q=(Q \cap D) D[S]=(Q \cap$ $D)_{v} D[S]=((Q \cap D) D[S])_{v}=Q_{v}$.

Case 2. $Q \cap D=0$. Then $Q K[S]$ is a maximal $t$-ideal of $K[S]$ by Lemma 3(2). If $Q K[S] \cap N=\emptyset$, then $Q K[S]$ is a height-one $t$-invertible prime ideal of $K[S]$ by Lemma $2(3)$; so $Q_{v} \subseteq Q_{v} K[S] \subseteq\left(Q_{v} K[S]\right)_{v}=$ $(Q K[S])_{v}=Q K[S]$. Thus $Q_{v} \subseteq Q K[S] \cap D[S]=Q$, and hence $Q_{v}=Q$. If $Q K[S] \cap N \neq \emptyset$, then $Q K[S]=K[J]$ for some maximal $t$-ideal $J$ of $S$ by Lemma 2(1). Thus $Q_{v} \subseteq Q_{v} K[S] \subseteq\left(Q_{v} K[S]\right)_{v}=(Q K[S])_{v}=$ $(K[J])_{v}=K\left[J_{v}\right]=K[J]=Q ;$ so $Q_{v}=Q$.

In [10, Proposition 5.5], M.H. Park shows that if $D$ is a strong Mori domain and if $G$ is a torsion-free abelian group of type $(0,0,0, \ldots)$, then $D[G]$ is an H-domain. It is well-known that a strong Mori domain is an H-domain. Thus the following corollary is a generalization of Park's results [10, Propositions 4.2 and 5.5].

Corollary 6. Let $D$ be an integral domain and $G$ be a torsion-free abelian group of type $(0,0,0, \ldots)$. Then $D[G]$ is an H-domain if and only if $D$ is an H-domain. 
Corollary 7. ([10, Proposition 4.2]) Let $\left\{X_{\alpha}\right\}$ be a nonempty set of indeterminates over $D$. Then $D$ is an H-domain if and only if $D\left[\left\{X_{\alpha}\right\}\right]$ is an H-domain.

Proof. Let $S=\sum_{\alpha}\left(\mathbb{Z}_{+}\right)_{\alpha}$, where $\left(\mathbb{Z}_{+}\right)_{\alpha}$ is the additive semigroup of nonnegative integers. Then $D[S]=D\left[\left\{X_{\alpha}\right\}\right]$ and $S$ is a torsion-free grading monoid whose quotient group is of type $(0,0,0, \ldots)$. Thus, $D$ is an H-domain if and only if $D\left[\left\{X_{\alpha}\right\}\right]$ is an H-domain by Theorem 5 .

We end this paper with an example which shows that in Theorem 5 , the assumption that $G$ is of type $(0,0,0, \ldots)$ is necessary.

EXAMPLE 8 . Let $\mathbb{R}$ be the field of real numbers and $\Gamma$ be the additive group of rational numbers.

(1) $\Gamma$ is a torsion-free abelian group.

(2) $\Gamma$ is not of type $(0,0,0, \ldots)$.

(3) $\mathbb{R}[\Gamma]$ is a $G C D$-domain, but not a $U F D$.

(4) Each maximal $t$-ideal of $\Gamma$ is a $v$-ideal.

(5) $\mathbb{R}[\Gamma]$ has the (Krull) dimension one.

(6) $\mathbb{R}[\Gamma]$ is not an H-domain.

Proof. (1) Clear. (2) This follows, because we have an infinite sequence of cyclic subgroups of $\Gamma$, say, $\left(\frac{1}{2}\right) \subsetneq\left(\frac{1}{2^{2}}\right) \subsetneq\left(\frac{1}{2^{3}}\right) \subsetneq \cdots$. (3) This is an immediate consequence of (1), (2) and [4, Theorems 14.5 and 14.16]. (4) Clear. (5) This follows from [4, Theorem 17.1]. (6) Let $Q$ be a maximal $t$-ideal of $\mathbb{R}[\Gamma]$ such that $Q^{-1} \supsetneq \mathbb{R}[\Gamma]$. Choose $u \in Q^{-1} \backslash \mathbb{R}[\Gamma]$. Then $\mathbb{R}[\Gamma] \subsetneq(1, u) \subseteq Q^{-1}$, and since $Q$ is a maximal $t$-ideal, we have $Q=Q_{v}=(1, u)^{-1}$. Since $\mathbb{R}[\Gamma]$ is a GCD-domain, $Q=(1, u)^{-1}$ must be principal. So by (3) and (5), there is a maximal $t$-ideal $Q$ of $\mathbb{R}[\Gamma]$ with $Q^{-1}=\mathbb{R}[\Gamma]$. Thus, $\mathbb{R}[\Gamma]$ is not an H-domain.

\section{Acknowledgements}

The author would like to thank the referee for several helpful comments.

\section{References}

[1] D.F. Anderson and G.W. Chang, Homogeneous splitting sets of a graded integral domain, J. Algebra 288 (2005), 527-544.

[2] S. El Baghdadi, L. Izelgue, and S. Kabbaj, On the class group of a graded domain, J. Pure Appl. Algebra 171 (2002), 171-184.

[3] R. Gilmer, Multiplicative Ideal Theory, Dekker, New York, 1972. 
[4] R. Gilmer, Commutative Semigroup Rings, The Univ. of Chicago, Chicago, 1984.

[5] R. Gilmer and T. Parker, Divisibility properties in semigroup rings, Michigan Math. J. 21 (1974), 65-86.

[6] S. Glaz and W.V. Vasconcelos, Flat ideals II, Manuscripta Math. 22 (1977), 325-341.

[7] F. Halter-Koch, Ideal Systems: An Introduction to Multiplicative Ideal Theory, Dekker, New York, 1998.

[8] E. Houston and M. Zafrullah, Integral domains in which each t-ideal is divisorial, Michigan Math. J. 35 (1988), 291-300.

[9] B.G. Kang, Prüfer v-multiplication domains and the ring $R[X]_{N_{v}}$, J. Algebra 123 (1989), 151-170.

[10] M.H. Park, Group rings and semigroup rings over strong Mori domains, J. Pure Appl. Algebra 163 (2001), 301-318.

[11] M. Zafrullah, Putting t-invertibility to use, in Non-Noetherian Commutative Ring Theory, Math. Appl. Kluwer Acad. Publ. Dordrecht 520 (2000), 429-457.

Department of Mathematics

University of Incheon

Incheon 406-772, Korea

E-mail: whan@incheon.ac.kr 